\title{
THE IDENTIFICATION AND UBV PHOTOMETRY \\ OF THE VISIBLE COMPONENT \\ OF THE CENTAURUS X-3 BINARY SYSTEM
}

\section{W. KRZEMIŃSK I}

Institute of Astronomy, Warsaw, Poland, and European Southern Observatory, La Silla, Chile

\begin{abstract}
The optical component of the pulsating binary X-ray source Cen X-3 is identified with a faint, $\langle V\rangle=13.35$, heavily reddened variable star lying slightly outside the 3rd Uhuru Catalogue error box. It shows a double peaked light curve with an amplitude of about $0.12 \mathrm{mag}$. which agrees both in period and phase with the X-ray data. The shallower minimum coincides in time with the X-ray pulsar eclipse. Interpretation of the light curve leads to the conclusion that the contribution from gravity darkening dominates to a large extent the reflection effect. The estimated distance to Cen $\mathrm{X}-3$ is about $8 \mathrm{kpc}$.
\end{abstract}

(This paper has been published in Astrophys. J. 192 (1974), L135. 